[Chem. Pharm. Bull.

32( 6 )2091-2099(1984) $]$

\title{
Studies on ${ }^{13}$ C Magnetic Resonance Spectroscopy. XVII. ${ }^{1)}$ Elucidation of Substituent-Induced Chemical Shifts of Substituted $n$-Alkane Series by Means of a Novel Substituent Entropy Constant $\sigma_{s^{\circ}}$
}

\author{
Su-Lan Hsiu, Hitoshi TaKai and Yoshio SaSAKI* \\ Faculty of Pharmaceutical Science, Osaka University, \\ 1-6 Yamadaoka, Suita 565, Japan
}

(Received July 27, 1983)

\begin{abstract}
Substituent effects on the ${ }^{13} \mathrm{C}$ and ${ }^{1} \mathrm{H}$ nuclear magnetic resonance (NMR) chemical shifts of aliphatic compounds have been studied. A good agreement was found between chemical shifts in the gas phase and those in $\mathrm{CCl}_{4}$ solution. Regression analyses of ${ }^{13} \mathrm{C}$ and ${ }^{1} \mathrm{H}$ chemical shifts in $\mathrm{CCl}_{4}$ solution and in the gas phase were carried out by using $\sigma_{i}, \sigma_{s^{\circ}}$ and $\sigma_{s \mathrm{MA}_{\mathrm{MA}}}$ as descriptors for $\alpha$-substituted butane and propane derivatives. Linear combinations of $\sigma_{i}, \sigma_{s^{\circ}}{ }^{+}$and/or $\left(\sigma_{s}^{+}\right)^{2}$, where the superscript " + " indicates an electron-donating substituent, are necessary for all ${ }^{13} \mathrm{C}$ and $\alpha-{ }^{1} \mathrm{H}$ chemical shifts of propane derivatives, but those of the $\beta$ - and $\gamma$-positions are correlated with $\sigma_{i}$. Except for the $\beta$-position signals, a term expressed as $\sigma_{\text {sim }}$ is needed to take account of the magnetic anisotropic effect of $\mathrm{Cl}, \mathrm{Br}, \mathrm{I}$ and $\mathrm{CN}$ groups on the ${ }^{13} \mathrm{C}$ and ${ }^{1} \mathrm{H}$ chemical shifts.
\end{abstract}

Keywords-_ $-{ }^{13} \mathrm{C}$ NMR; ${ }^{1} \mathrm{H}$ NMR; substituent entropy constant $\sigma_{s}$; gas phase NMR; substituent constant; $\alpha$-substituted propane; $\alpha$-substituted butane; substituent effect

Three kinds of approaches, namely, empirical, semi-empirical and non-empirical, are generally used for the elucidation of the substituent-induced chemical shifts (SCS) of aliphatic, as well as aromatic series of compounds. Since the quantum chemical treatment of nuclear magnetic resonance (NMR) chemical shifts (CS) was established by Pople, ${ }^{2)}$ several kinds of theoretical molecular orbital calculation have been applied for estimation of chemical shifts, ${ }^{3,4)}$ but these non-empirical approaches are not always effective. Although the additivity rule was found to be useful to predict the SCS of some families such as substituted alkanes ${ }^{5}$ and benzene derivatives, ${ }^{6}$ ) this empirical method has limited applicability. In our previous report $^{7)}$ on a semi-empirical derivation of the SCS of substituted benzene and naphthalene series, the total charge densities obtained by the MINDO/2 method could not be successfully correlated with the observed SCS of the ipso-position. These results prompted us to analyze this subject by using an empirical descriptor based on the thermodynamic concept, because the quantum chemical approach does not always represent the intrinsic thermodynamic information.

Recently, we proposed the novel substituent entropy constant $\sigma_{s^{\circ}}{ }^{8)}$ defined as $\sigma_{s^{\circ}}=$ $\log \left(S_{\mathrm{R}}^{\circ} / S_{\mathrm{H}}^{\circ}\right)$ for numerous kinds of substituent groups, and confirmed that this descriptor is very useful. ${ }^{9)}$ In this work, the regression analyses of ${ }^{13} \mathrm{C} \mathrm{SCS}$ of substituted $n$-butanes as well as those of ${ }^{1} \mathrm{H}$ SCS of substituted $n$-propanes were carried out by using the novel descriptor $\left.\sigma_{s^{\circ}},{ }^{8}\right)$ together with the Hammett-type substituent constant $\sigma_{i},{ }^{10)}$ including the contributions of the magnetic anisotropy and electric field effects. The $\sigma_{i}$ scale is related to the Taft scale by $\sigma_{i}=0.74 \sigma_{\mathrm{I}}{ }^{11)}$

\section{Experimental}

${ }^{13} \mathrm{C}$ CS of Substituted $n$-Propane (PrR) and $n$-Butane (BuR) Derivatives- $-{ }^{13} \mathrm{C} \mathrm{SCS}$ of PrR were measured in the 
vapor phase at $170^{\circ} \mathrm{C}$ using a specially machined sample tube (Chart 1), by a substitution method. The resonance signal for $\mathrm{C}_{6} \mathrm{H}_{5} \cdot \mathrm{Et}$ as a reference was obtained and then $\mathrm{C}_{6} \mathrm{H}_{5} \cdot \mathrm{Et}$ was replaced by the sample. The resonance signal of the sample was obtained, then the reference was again placed in the probe to confirm the stability of the field. The SCS values of $\mathrm{BuR}$ were determined in $\mathrm{CCl}_{4}(0.2 \mathrm{M})$ with $\mathrm{Me}_{4} \mathrm{Si}$ as an internal reference. Observed values in the two series are summarized in Table $\mathrm{I}$.

${ }^{1} \mathbf{H}$ CS and ${ }^{1} \mathbf{H}$ SCS of Substituted $\boldsymbol{n}$-Propanes (PrR)—_References used for determinations of PrR in the gas phase at $100^{\circ} \mathrm{C}$ as well as in $\mathrm{CCl}_{4}$ solution $(0.1 \mathrm{M})$ were the same as described above, and the observed values are summarized in Table II.

Method of Regression Analysis__-Multiple regression analyses were carried out by using an ACOS 900 system

TABLE I. ${ }^{13} \mathrm{C} \mathrm{SCS}$ of BuR and ${ }^{13} \mathrm{C} \mathrm{CS}^{a)}$ of PrR (ppm)

\begin{tabular}{|c|c|c|c|c|c|c|}
\hline \multirow{2}{*}{$\begin{array}{c}\text { Substituent } \\
\text { (R) }\end{array}$} & \multicolumn{3}{|c|}{$\mathrm{BuR}\left(\mathrm{In} \mathrm{CCl}_{4}\right)$} & \multicolumn{3}{|c|}{ PrR (Gas phase) } \\
\hline & $\alpha$ & $\beta$ & $\gamma$ & $\alpha$ & $\beta$ & $\gamma$ \\
\hline $\mathrm{NMe}_{2}$ & 40.10 & 5.38 & -4.12 & - & - & - \\
\hline $\mathrm{NH}_{2}$ & 27.84 & 10.89 & -4.90 & 13.96 & -3.30 & -21.43 \\
\hline $\mathrm{OEt}$ & 56.24 & 7.13 & -5.39 & - & - & - \\
\hline $\mathrm{OMe}$ & 44.28 & 6.93 & -5.48 & 26.09 & -7.44 & -22.00 \\
\hline $\mathrm{OH}$ & 48.15 & 10.13 & -5.87 & 34.07 & -4.11 & -22.42 \\
\hline tert-Bu & 30.23 & 2.04 & -1.20 & 17.16 & -12.72 & -17.35 \\
\hline iso-Pr & 24.96 & 4.86 & -1.89 & 11.77 & -10.07 & -18.13 \\
\hline Et & 17.76 & 6.74 & -2.19 & - & -8.30 & -18.61 \\
\hline $\mathrm{Me}$ & 8.51 & 9.29 & -2.51 & - & - & - \\
\hline $\mathbf{F}$ & 70.10 & 8.00 & -6.70 & - & - & - \\
\hline $\mathrm{Cl}$ & 29.87 & 9.80 & -4.82 & 13.81 & -4.20 & -20.98 \\
\hline $\mathrm{Br}$ & 18.40 & 9.89 & -3.50 & 0.90 & -3.81 & -19.36 \\
\hline I & -8.66 & 10.64 & -1.20 & -28.95 & -3.15 & -17.26 \\
\hline $\mathrm{H}^{b)}$ & 0 & 0 & 0 & - & - & - \\
\hline $\mathrm{COPh}$ & 24.00 & 1.45 & -2.33 & - & - & 一 \\
\hline $\mathrm{COEt}$ & 27.90 & 1.09 & -2.45 & 13.42 & -13.04 & -18.55 \\
\hline COMe & 29.20 & 0.95 & -2.54 & 14.74 & -13.04 & -18.61 \\
\hline $\mathrm{CHO}$ & 29.54 & -0.67 & -2.55 & 15.52 & -14.78 & -18.67 \\
\hline $\mathrm{CONH}_{2}$ & 22.05 & 3.00 & -2.27 & - & - & - \\
\hline COOEt & 19.86 & 2.18 & -2.56 & 5.82 & -11.98 & -18.70 \\
\hline COOMe & 19.52 & 2.11 & -2.59 & 5.33 & -12.04 & -18.76 \\
\hline $\mathrm{CN}$ & 2.89 & 2.68 & -3.02 & -10.85 & -12.23 & -19.33 \\
\hline $\mathrm{NO}_{2}$ & 61.00 & 4.44 & -5.24 & - & -9.53 & -21.42 \\
\hline
\end{tabular}

a) ${ }^{13} \mathrm{C}$ chemical shift relative to $\mathrm{CH}_{2}$ of neat $\mathrm{C}_{6} \mathrm{H}_{5} \cdot \mathrm{Et}$.

b) ${ }^{13} \mathrm{C}$ chemical shifts from internal TMS at $\alpha$-, $\beta$ - and $\gamma$-positions are $13.60,24.62$ and $24.62 \mathrm{ppm}$, respectively.

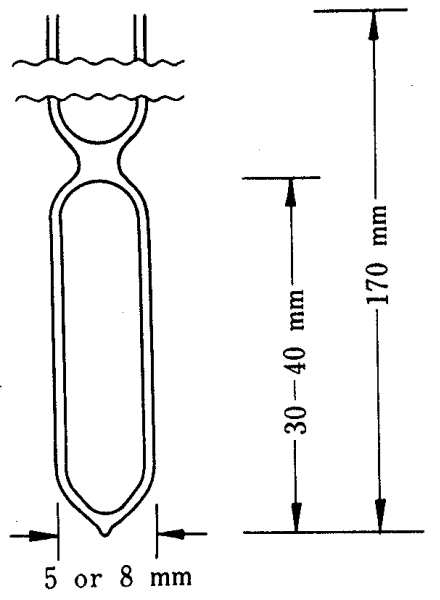

Chart 1. Specially Machined Sample Tube Used for Determination in the Gas Phase 
TABLE II. ${ }^{1} \mathrm{H} \mathrm{CS}^{a}$ in Gas Phase and ${ }^{1} \mathrm{H} \mathrm{SCS}$ in $\mathrm{CCl}_{4}$ of $\operatorname{PrR}(\mathrm{ppm})$

\begin{tabular}{|c|c|c|c|c|c|c|}
\hline \multirow{2}{*}{$\begin{array}{l}\text { Substituent } \\
\text { (R) }\end{array}$} & \multicolumn{3}{|c|}{ Gas phase } & \multicolumn{3}{|c|}{ In $\mathrm{CCl}_{4}$} \\
\hline & $\alpha$ & $\beta$ & $\gamma$ & $\alpha$ & $\beta$ & $\gamma$ \\
\hline $\mathrm{NH}_{2}$ & 0.59 & -0.64 & -1.18 & 1.74 & 0.06 & 0.05 \\
\hline $\mathrm{OPr}$ & - & - & - & 2.43 & 0.18 & 0.06 \\
\hline $\mathrm{OEt}$ & - & - & - & 2.42 & 0.18 & 0.05 \\
\hline $\mathrm{OMe}$ & 1.18 & -0.51 & -1.14 & 2.37 & 0.18 & 0.05 \\
\hline $\mathrm{OH}$ & 1.46 & -0.54 & -1.15 & 2.64 & 0.18 & 0.08 \\
\hline tert-Bu & -0.83 & -0.96 & -1.20 & 0.35 & -0.20 & 0.04 \\
\hline $\mathrm{Et}$ & -0.72 & -0.72 & -1.17 & 0.42 & -0.09 & 0.03 \\
\hline $\mathrm{Cl}$ & 1.21 & -0.37 & -1.10 & 2.57 & 0.46 & 0.19 \\
\hline $\mathrm{Br}$ & 1.12 & -0.26 & -1.08 & 2.45 & 0.52 & 0.19 \\
\hline I & 0.95 & -0.30 & -1.13 & 2.28 & 0.53 & 0.18 \\
\hline $\mathrm{H}^{b)}$ & - & - & - & 0 & 0 & 0 \\
\hline $\mathrm{COPh}$ & - & - & - & 2.02 & 0.40 & 0.15 \\
\hline COEt & 0.12 & -0.44 & -1.17 & 1.49 & 0.22 & 0.05 \\
\hline $\mathrm{COMe}$ & 0.17 & -0.46 & -1.19 & 1.48 & 0.25 & 0.07 \\
\hline $\mathrm{CHO}$ & 0.11 & -0.43 & -1.13 & 1.49 & 0.31 & 0.12 \\
\hline $\mathrm{CONH}_{2}$ & - & - & - & 1.25 & 0.28 & 0.11 \\
\hline COOEt & 0.08 & -0.43 & -1.14 & 1.36 & 0.26 & 0.11 \\
\hline COOMe & 0.10 & -0.43 & -1.14 & 1.37 & 0.28 & 0.11 \\
\hline $\mathrm{CN}$ & -0.05 & -0.51 & -1.05 & 1.43 & 0.36 & 0.26 \\
\hline $\mathrm{NO}_{2}$ & 2.01 & -0.13 & -1.10 & 3.38 & 0.69 & 0.19 \\
\hline
\end{tabular}

a) ${ }^{1} \mathrm{H}$ chemical shift relative to $\mathrm{Me}$ of neat $\mathrm{C}_{6} \mathrm{H}_{5} \cdot \mathrm{Et}$.

b) Chemical shifts from internal TMS at $\alpha-, \beta$ - and $\gamma$-positions are $0.85,1.36$ and $0.85 \mathrm{ppm}$, respectively. ${ }^{12)}$

computer at Osaka University Computer Center utilizing a library program, NEC TSS LIBRARY TSS/LIB-6. The standard deviation (SD) is given by $\mathrm{SD}=\left[S_{\mathrm{se}} /(n-k-1)\right]^{1 / 2}$, where $n$ and $k$ denote the number of observations and variables, and $S_{\text {se }}$ is the sum of squares of residuals.

Substituent Entropy Constant $\sigma_{s}{ }^{\circ}$ - The substituent entropy constants of $\operatorname{Pr} R$ and BuR ${ }^{13)}$ show good linear correlations with those of methane derivatives $(\mathrm{MeR})^{8)}$ as follows.

$$
\begin{aligned}
\sigma_{s^{\circ} \mathrm{PrR}}= & 0.755 \sigma_{s^{\circ} \mathrm{MeR}}-0.005 \\
r= & 0.996, \mathrm{SD}=0.004, n=16 \\
(\mathrm{R}= & \mathrm{NH}_{2}, \mathrm{OMe}, \mathrm{OH}, \text { tert }-\mathrm{Bu}, \text { iso-Pr, Et, Me, F, Cl, Br, I, H, Ph, COMe, } \\
& \left.\mathrm{CN}, \mathrm{NO}_{2}\right) \\
\sigma_{s^{\circ} \mathrm{BuR}}= & 0.668 \sigma_{s^{\circ} \mathrm{MeR}}-0.005 \\
r= & 0.996, \mathrm{SD}=0.004, n=11 \\
(\mathrm{R}= & \left.\mathrm{NH}_{2}, \mathrm{OH}, \text { tert }-\mathrm{Bu}, \text { iso-Pr, Et, } \mathrm{Me}, \mathrm{Cl}, \mathrm{Br}, \mathrm{H}, \mathrm{Ph}, \mathrm{NO}_{2}\right)
\end{aligned}
$$

The substituent entropy constants of MeR instead of those of $\operatorname{PrR}$ and BuR could be used in the regression analyses. By dividing by the factors 0.755 and 0.668 , all the coefficients of the entropic terms written in the regression equations can be converted to $\sigma_{s^{\circ} \mathrm{PrR}}$ and $\sigma_{s^{\circ} \mathrm{BuR}}$.

\section{Results and Discussion}

\section{Quadratic Equation of $\sigma_{s^{\circ}}^{+}$}

In this work, the symbol $\sigma_{s^{\circ}}^{+}$is used to express the substituent entropy constants of an electron-donating group $(-\mathrm{R})$, and the numerical values of $\sigma_{s^{\circ}}^{+}$are identical with the $\sigma_{s^{\circ}}$ of that group, but for the electron-attracting group $(+\mathrm{R})$ those of $\sigma_{s^{\circ}}^{+}$are zero. When the SCS of the $-\mathrm{R}$ group are plotted against $\sigma_{s^{\circ}}{ }^{8)}$ a quadratic curve ( $c f$. Fig. 1) can be expected. In a regression analysis of the ${ }^{13} \mathrm{C} \mathrm{SCS}$ of the $\beta$-position of $n$-BuR, the correlation coefficient of 


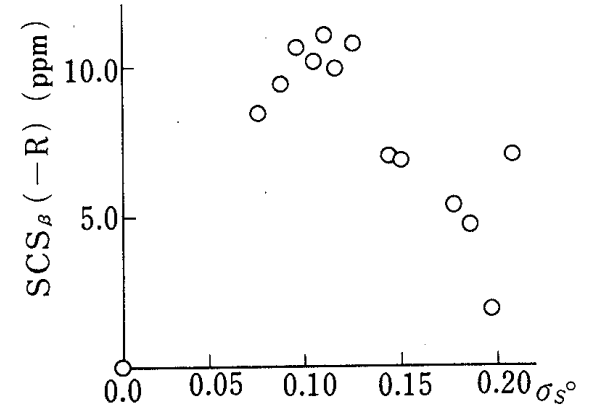

Fig. 1. Correlation between $\sigma_{s^{\circ}}$ and ${ }^{13} \mathrm{C} \mathrm{SCS}$ at the $\beta$-Position of BuR Bearing a $-\mathrm{R}$ Group

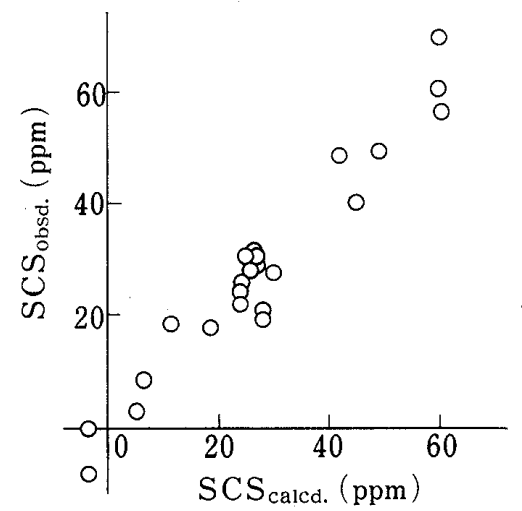

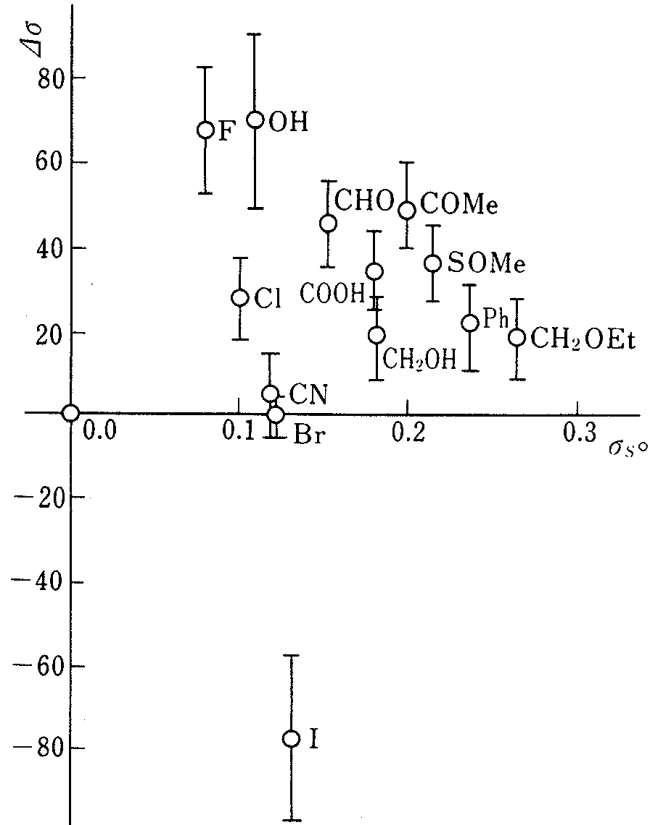

Fig. 2. Correlation between ${ }^{13} \mathrm{C}$ Shielding Anisotropies $\Delta \sigma$ and $\sigma_{s^{\circ}}$ of $\mathrm{MeR}$
Fig. 3. Correlation between Observed and Calculated SCS of $\operatorname{BuR}\left({ }^{13} \mathrm{C}-\alpha\right)$

$\operatorname{SCS}_{\text {calcd. }}=138.12 \sigma_{i}+175.53 \sigma_{s^{\circ}}^{+}$
$-3510.98 \sigma_{s_{\mathrm{MA}}^{\circ}} \beta^{2.16}$

$r=0.674$ obtained by using two descriptors, $\sigma_{i}$ and $\sigma_{s^{\circ}}^{+}$, could be improved to 0.937 on addition of the $\left(\sigma_{s^{\circ}}^{+}\right)^{2}$ term as a descriptor.

The choice of a quadratic equation of $\sigma_{s^{\circ}}$-in other words, the presence of entropy maxima of ${ }^{13} \mathrm{C} \mathrm{SCS}_{\alpha}$ and $\mathrm{SCS}_{\beta}$, as well as an entropy minimum of $\mathrm{SCS}_{\gamma}$ - suggests the existence of a most favorable or unfavorable thermodynamic state. The $\Delta S^{\circ}$ data given by Brown et al ${ }^{14)}$ for the thermal dissociation of amine-boron adduct and those determined in the dissociation of $\mathrm{R} \cdot \mathrm{CH}_{2} \mathrm{CO}_{2} \mathrm{H}$ in aqueous media ${ }^{15)}$ could be regarded as typical examples.

\section{Magnetic Anisotropy Effect in Regression Analysis}

To take account of the contribution of diamagnetic shielding to SCS, two parameters, namely, magnetic susceptibility $\chi$ and magnetic shielding $\sigma$, could be taken into account, where $\sigma$ and $\chi$ are represented by Eqs. 1 and 2. ${ }^{16)}$

$$
\begin{aligned}
& \sigma_{\text {avg }}^{\mathrm{d}}=\left(1 / 3 c^{2}\right)\langle 1 / \mathrm{r}\rangle \\
& \chi_{\text {avg }}^{\mathrm{d}}=\left(1 / 4 c^{2}\right)\left\langle\mathrm{r}^{2}\right\rangle
\end{aligned}
$$

where $c$ denotes the velocity of light, and $\langle\mathrm{r}\rangle$ means the average value of molecular radius.

In addition, the values of observed ${ }^{13} \mathrm{C}$ shielding anisotropy $\Delta \sigma$ of monosubstituted methanes determined experimentally ${ }^{17)}$ are assumed to be quadratic with respect to $\sigma_{s^{\circ}}$ as illustrated in Fig. 2, where the data for halogens $(\mathrm{Cl}, \mathrm{Br}, \mathrm{I})$ and $\mathrm{CN}$ gave another line bisecting the parabola. Consequently, an equation containing $-a\left(\sigma_{s^{\circ}}\right)^{2}+b \sigma_{s^{\circ}}+c$ includes the anisotropy $\Delta \sigma$, except for the 4 kinds of substituents written above. In this work, the additional contribution due to these 4 groups is expressed by the symbol $\sigma_{s_{\text {MA }}}$, corresponding to the square term $\left(\sigma_{s^{\circ}}\right)^{2}$ followed by Eq. 2 . Namely, the numerical values of $\sigma_{s_{\mathrm{MA}}}$ for halogens and 
$\mathrm{CN}$ are the same as the $\left(\sigma_{s^{\circ}}\right)^{2}$ values of the 4 groups.

\section{Electric Field Effect in Regression Analysis}

The component $E_{z}$ of the electric field effect is given by Eq. $3,{ }^{18)}$ having dimensions of $\left(\mathrm{erg} \cdot \mathrm{cm}^{-3}\right)^{1 / 2}$

$$
\mathrm{E}_{\mathrm{z}}=2 \mu \cos \theta / \mathrm{r}^{3}
$$

Ez could be divided into two parts, namely an enthalpic term expressed by $\sigma_{i}$ or $\sigma_{\pi}$, and an entropic term expressed by $\sigma_{s^{\circ}}$.

\section{Results of Regression Analyses}

The results of comparisons of the observed ${ }^{13} \mathrm{C}$ and ${ }^{1} \mathrm{H}$ chemical shifts in the gas phase with those in $\mathrm{CCl}_{4}$ solution are summarized in the following regression equations.

$$
\begin{aligned}
& (-\mathrm{R}): \text { electron-donating group } \\
& (+\mathrm{R}): \text { electron-attracting group } \\
& (\mathrm{T}): \text { sum of electron-donating and electron-attracting group }
\end{aligned}
$$

I-1. ${ }^{13} \mathrm{C} \mathrm{SCS}$ at $\alpha$-Position of BuR (in $\mathrm{CCl}_{4}$ ) (cf. Fig. 3)

$$
\begin{aligned}
(-\mathrm{R}): \mathrm{SCS}_{\alpha}= & 143.09 \sigma_{i}+138.68 \sigma_{s^{\circ}}-3640.34 \sigma_{\text {siA }}+2.16 \\
& ( \pm 11.19) \quad( \pm 23.58) \quad( \pm 277.94) \quad( \pm 3.87) \\
r= & 0.979, \mathrm{SD}=5.03, n=14, F=77.00 \\
(\mathrm{R}= & \left.\mathrm{NMe}_{2}, \mathrm{NH}_{2}, \mathrm{OEt}, \mathrm{OMe}, \mathrm{OH}, \text { tert }-\mathrm{Bu}, \text { iso-Pr, Et, Me, F, Cl, Br, I, H }\right) \\
(+\mathrm{R}): \mathrm{SCS}_{\alpha}= & 131.53 \sigma_{i}-3534.84 \sigma_{s_{\text {siA }}}-2.86 \\
& ( \pm 15.26) \quad( \pm 435.32) \quad( \pm 3.73) \\
r= & 0.965, \mathrm{SD}=4.99, n=10, F=46.70 \\
(\mathrm{R}= & \left.\mathrm{H}, \mathrm{COPh}, \mathrm{COEt}, \mathrm{COMe}, \mathrm{CHO}, \mathrm{CONH}_{2}, \mathrm{COOEt}, \mathrm{COOMe}, \mathrm{CN}, \mathrm{NO}_{2}\right) \\
(\mathrm{T}): \mathrm{SCS}_{\alpha}= & 138.12 \sigma_{i}+175.53 \sigma_{s^{\circ}}^{+}-3510.98 \sigma_{\text {sinA }}-3.49 \\
& ( \pm 9.99) \quad( \pm 16.20) \quad( \pm 238.68) \quad( \pm 2.77) \\
r= & 0.967, \mathrm{SD}=5.23, n=23, F=91.46
\end{aligned}
$$

I-2. ${ }^{13} \mathrm{C} \mathrm{CS}$ at $\alpha$-Position of PrR (Gas Phase)

$$
\begin{aligned}
(\mathrm{T}): \mathrm{CS}_{\alpha}= & 128.67 \sigma_{i}+178.32 \sigma_{s^{-}}^{+}-3564.78 \sigma_{s \mathrm{MA}}-16.89 \\
& ( \pm 32.27) \quad( \pm 41.92) \quad( \pm 522.45) \quad( \pm 8.11) \\
r= & 0.928, \mathrm{SD}=6.52, n=14, F=20.68 \\
(\mathrm{R}= & \mathrm{NH}_{2}, \mathrm{OMe}, \mathrm{OH}, \text { tert }-\mathrm{Bu}, \text { iso-Pr, } \mathrm{Cl}, \mathrm{Br}, \mathrm{I}, \mathrm{COEt}, \mathrm{COMe}, \mathrm{CHO}, \mathrm{COOEt}, \\
& \mathrm{COOMe}, \mathrm{CN})
\end{aligned}
$$

In our previous report ${ }^{19)}$ of this series, the torsion barrier $V$ determined on the MeR series $\left(\mathrm{R}=\right.$ electron-donating substituent) was found to be dependent on $\Delta S_{+}^{\circ}$. The relation expressed by Eq. 6 suggests that the torsion barrier is well defined by $\sigma_{s^{\circ}}^{+}$.

$$
\begin{aligned}
V= & 17.18 \sigma_{s^{\circ}}^{+}+0.42 \\
& ( \pm 2.28)( \pm 0.29) \\
r= & 0.915, \mathrm{SD}=0.67, n=13, F=56.92
\end{aligned}
$$

I-3. ${ }^{1} \mathrm{H} \mathrm{SCS}$ at $\alpha$-Position of PrR (in $\mathrm{CCl}_{4}$ )

$$
\begin{aligned}
(-\mathrm{R}): \mathrm{SCS}_{\alpha}= & 8.30 \sigma_{i}+9.23 \sigma_{s^{\circ}}-22.98\left(\sigma_{s^{\circ}}\right)^{2}-68.51 \sigma_{\sin }+0.02 \\
& ( \pm 0.77) \quad( \pm 3.32) \quad( \pm 11.35) \quad( \pm 16.69) \quad( \pm 0.24) \\
r= & 0.983, \mathrm{SD}=0.24, n=11, F=42.21 \\
(\mathrm{R}= & \left.\mathrm{NH}_{2}, \mathrm{OPr}, \mathrm{OEt}, \mathrm{OMe}, \mathrm{OH}, \text { tert }-\mathrm{Bu}, \mathrm{Et}, \mathrm{Cl}, \mathrm{Br}, \mathrm{I}, \mathrm{H}\right)
\end{aligned}
$$




$$
\begin{aligned}
(+\mathrm{R}): \mathrm{SCS}_{\alpha}= & 7.23 \sigma_{i}-111.63 \sigma_{s \mathrm{AA}}-0.04 \\
& ( \pm 0.86) \quad( \pm 24.43) \quad( \pm 0.21) \\
r= & 0.954, \mathrm{SD}=0.28, n=10, F=35.69
\end{aligned}
$$

$\left(\mathrm{R}=\mathrm{H}, \mathrm{COPh}, \mathrm{COEt}\right.$, COMe, $\mathrm{CHO}, \mathrm{CONH}_{2}, \mathrm{COOEt}, \mathrm{COOMe}, \mathrm{CN}, \mathrm{NO}_{2}$ )

( T): $\operatorname{SCS}_{\alpha}=7.73 \sigma_{i}+14.02 \sigma_{s^{\circ}}^{+}-37.75\left(\sigma_{s^{\circ}}^{+}\right)^{2}-77.27 \sigma_{\operatorname{sinA}}-0.20$

$$
\begin{array}{lllll}
( \pm 0.71) & ( \pm 2.74) \quad( \pm 11.57) \quad( \pm 16.31) & ( \pm 0.19)
\end{array}
$$

$$
r=0.947, \mathrm{SD}=0.31, n=20, F=32.70
$$

\section{I-4. ${ }^{1} \mathrm{H}$ CS at $\alpha$-Position of PrR (Gas Phase)}

$$
\begin{aligned}
(\mathrm{T}): \mathrm{CS}_{\alpha}= & 7.59 \sigma_{i}+21.37 \sigma_{s^{\circ}}^{+}-75.05\left(\sigma_{s^{\circ}}^{+}\right)^{2}-87.69 \sigma_{s_{\mathrm{MA}}}-1.62 \\
& ( \pm 0.90) \quad( \pm 3.21) \quad( \pm 19.63) \quad( \pm 15.08) \quad( \pm 0.26) \\
r= & 0.964, \mathrm{SD}=0.25, n=15, F=32.72 \\
(\mathrm{R}= & \mathrm{NH}_{2}, \mathrm{OMe}, \mathrm{OH}, \text { tert }-\mathrm{Bu}, \mathrm{Et}, \mathrm{Cl}, \mathrm{Br}, \mathrm{I}, \mathrm{COEt}, \mathrm{COMe}, \mathrm{CHO}, \mathrm{COOEt}, \mathrm{COOMe}, \\
& \left.\mathrm{CN}, \mathrm{NO}_{2}\right)
\end{aligned}
$$

\section{II-1. ${ }^{13} \mathrm{C} \mathrm{SCS}$ at $\beta$-Position of $\mathrm{BuR}$ (in $\mathrm{CCl}_{4}$ )}

The previous result ${ }^{19)}$

$$
\begin{aligned}
& \mathrm{SCS}_{\beta}=1.46 \Delta S_{+}^{\circ}-0.05\left(\Delta S_{+}^{\circ}\right)^{2}-0.02 \\
& \text { was revised to Eq. } 9 \text { in this work. } \\
& (-\mathrm{R}): \mathrm{SCS}_{\beta}=0.86 \sigma_{i}+170.31 \sigma_{s^{\circ}}-754.51\left(\sigma_{s^{\circ}}\right)^{2}-0.02 \\
& ( \pm 2.35) \quad( \pm 19.66) \quad( \pm 87.54) \quad( \pm 1.00) \\
& r=0.965, \mathrm{SD}=1.04, n=13, F=40.67 \\
& \left(\mathrm{R}=\mathrm{NMe}_{2}, \mathrm{NH}_{2}, \mathrm{OMe}, \mathrm{OH} \text {, tert-Bu, iso-Pr, Et, } \mathrm{Me}, \mathrm{F}, \mathrm{Cl}, \mathrm{Br}, \mathrm{I}, \mathrm{H}\right) \\
& (+\mathrm{R}): \mathrm{SCS}_{\beta}=8.61 \sigma_{i}-0.25 \\
& ( \pm 1.57) \quad( \pm 0.44) \\
& r=0.913, \mathrm{SD}=0.59, n=8, F=30.22 \\
& \left(\mathrm{R}=\mathrm{H}, \mathrm{COPh}, \mathrm{COEt} \text {, COMe, COOEt, COOMe, } \mathrm{CN}, \mathrm{NO}_{2}\right) \\
& \text { ( T): } \operatorname{SCS}_{\beta}=4.18 \sigma_{i}+143.25 \sigma_{s^{\circ}}^{+}-638.20\left(\sigma_{s^{\circ}}^{+}\right)^{2}+0.82 \\
& ( \pm 1.77) \quad( \pm 10.09) \quad( \pm 56.76) \quad( \pm 0.57) \\
& r=0.968, \mathrm{SD}=1.02, n=20, F=80.30
\end{aligned}
$$

II-2. ${ }^{13} \mathrm{C} \mathrm{CS}$ at $\beta$-Position of PrR (Gas Phase)

( T): $\mathrm{CS}_{\beta}=5.96 \sigma_{i}+148.83 \sigma_{s^{\circ}}^{+}-672.23\left(\sigma_{s^{\circ}}^{+}\right)^{2}-13.71$

$$
( \pm 2.93) \quad( \pm 13.00) \quad( \pm 79.68) \quad( \pm 0.98)
$$

$r=0.968, \mathrm{SD}=1.12, n=15, F=54.26$

$\left(\mathrm{R}=\mathrm{NH}_{2}, \mathrm{OMe}, \mathrm{OH}\right.$, tert $-\mathrm{Bu}$, iso- $\mathrm{Pr}, \mathrm{Et}, \mathrm{Cl}, \mathrm{Br}, \mathrm{I}, \mathrm{COEt}, \mathrm{COMe}$, COOEt, COOMe, $\mathrm{CN} ; \mathrm{NO}_{2}$ )

II-3. ${ }^{1} \mathrm{H} \mathrm{SCS}$ at $\beta$-Position of $\operatorname{PrR}$ (in $\mathrm{CCl}_{4}$ )

$$
\begin{aligned}
(-\mathrm{R}): \mathrm{SCS}_{\beta}= & 1.62 \sigma_{i}-0.07 \\
& ( \pm 0.15) \quad( \pm 0.03) \\
r= & 0.964, \mathrm{SD}=0.07, n=11, F=118.43 \\
(\mathrm{R}= & \text { the same as those included in } \mathrm{I}-3) \\
(+\mathrm{R}): \mathrm{SCS}_{\beta}= & 1.48 \sigma_{i}-1.97 \sigma_{\sin }-0.02 \\
& ( \pm 0.20) \quad( \pm 0.66) \quad( \pm 0.05) \\
r= & 0.943, \mathrm{SD}=0.07, n=10, F=27.83
\end{aligned}
$$


( $R=$ the same as those included in $\mathrm{I}-3$ )

$$
\begin{aligned}
(\mathrm{T}): \mathrm{SCS}_{\beta}= & 1.63 \sigma_{i}-18.20 \sigma_{s \mathrm{MA}}-0.07 \\
& ( \pm 0.12) \quad( \pm 5.07) \quad( \pm 0.03) \\
r= & 0.959, \mathrm{SD}=0.06, n=20, F=96.90
\end{aligned}
$$

II-4. $\quad{ }^{1} \mathrm{H} \mathrm{CS}$ at $\beta$-Position of PrR (Gas Phase)

$$
\begin{aligned}
(\mathrm{T}): \mathrm{CS}_{\beta}= & 1.40 \sigma_{i}-23.89 \sigma_{\text {siAA }}-0.76 \\
& ( \pm 0.12) \quad( \pm 4.66) \quad( \pm 0.03) \\
r= & 0.962, \mathrm{SD}=0.06, n=15, F=73.88 \\
(\mathrm{R}= & \text { the same as those included in } \mathrm{I}-4) .
\end{aligned}
$$

III-1. ${ }^{13} \mathrm{C} \mathrm{SCS}$ at $\gamma$-Position of BuR (in $\mathrm{CCl}_{4}$ )

$$
\begin{aligned}
& (-\mathrm{R}): \mathrm{SCS}_{\gamma}=-13.54 \sigma_{i}-44.07 \sigma_{s^{\circ}}+154.71\left(\sigma_{s^{\circ}}\right)^{2}+298.59 \sigma_{\operatorname{sinA}}-0.02 \\
& \begin{array}{lllll}
( \pm 1.15) & ( \pm 8.27)( \pm 33.63)( \pm 27.36) \quad( \pm 0.48)
\end{array} \\
& r=0.980, \mathrm{SD}=0.49, n=14, F=55.44 \\
& (\mathrm{R}=\text { the same as those included in } \mathrm{I}-1) \\
& \begin{aligned}
(+R): \operatorname{SCS}_{\gamma}= & -11.37 \sigma_{i}+126.60 \sigma_{\operatorname{sinA}}-0.02 \\
& ( \pm 0.16)( \pm 4.46)( \pm 0.04)
\end{aligned} \\
& r=0.999, \mathrm{SD}=0.05, n=10, F=2686.77 \\
& \text { ( } R=\text { the same as those included in } I-1) \\
& \text { ( T): } \mathrm{SCS}_{\gamma}=-12.82 \sigma_{i}-44.30 \sigma_{s^{\circ}}^{+}+151.84\left(\sigma_{s^{\circ}}^{+}\right)^{2}+249.67 \sigma_{\text {si }}+0.15 \\
& \begin{array}{lllll}
( \pm 1.02) & ( \pm 5.02)( \pm 25.39) & ( \pm 25.20) & ( \pm 0.28)
\end{array} \\
& r=0.960, \mathrm{SD}=0.53, n=23, F=53.09
\end{aligned}
$$

III-2. ${ }^{13} \mathrm{C} \mathrm{CS}$ at $\gamma$-Position of PrR (Gas Phase)

( T): $\mathrm{CS}_{\gamma}=-12.43 \sigma_{i}-53.11 \sigma_{s^{+}}^{+}+206.83\left(\sigma_{s^{\circ}}^{+}\right)^{2}+267.59$

$r=0.930, \mathrm{SD}=0.70, n=16, F=17.63$

$\left(\mathrm{R}=\mathrm{NH}_{2}, \mathrm{OMe}\right.$, OH, tert $-\mathrm{Bu}$, iso- $\mathrm{Pr}, \mathrm{Et}, \mathrm{Cl}, \mathrm{Br}, \mathrm{I}, \mathrm{COEt}$, COMe, CHO, COOEt, COOMe, $\mathrm{CN}, \mathrm{NO}_{2}$ )

\section{III-3. ${ }^{1} \mathrm{H} \mathrm{SCS}$ at $\gamma$-Position of PrR (in $\mathrm{CCl}_{4}$ )}

$$
\begin{aligned}
& (-\mathrm{R}): \mathrm{SCS}_{\gamma}=0.20 \sigma_{i}+6.20 \sigma_{\text {siAA }}+0.03 \\
& ( \pm 0.06) \quad( \pm 1.33) \quad( \pm 0.01) \\
& r=0.964, \mathrm{SD}=0.02, n=11, F=52.32 \\
& \text { ( } R=\text { the same as those included in } \mathrm{I}-3 \text { ) } \\
& (+\mathrm{R}): \mathrm{SCS}_{\gamma}=0.40 \sigma_{i}+5.56 \sigma_{\text {si A }}+0.01 \\
& ( \pm 0.10) \quad( \pm 2.82) \quad( \pm 0.02) \\
& r=0.919, \mathrm{SD}=0.03, n=10, F=19.10 \\
& \text { ( } R=\text { the same as those included in I-3) } \\
& \text { ( T): } \mathrm{SCS}_{\gamma}=0.34 \sigma_{i}+4.41 \sigma_{\text {si AA }}+0.02 \\
& ( \pm 0.06) \quad( \pm 1.32) \quad( \pm 0.01) \\
& r=0.915, \mathrm{SD}=0.03, n=20, F=43.44
\end{aligned}
$$

III-4. ${ }^{1} \mathrm{H}$ CS at $\gamma$-Position of PrR (Gas Phase)

$$
\begin{aligned}
(\mathrm{T}): \mathrm{SC}_{\gamma}= & 0.16 \sigma_{i}+3.71 \sigma_{\mathrm{sinA}}-1.18 \\
& ( \pm 0.04) \quad( \pm 1.08) \quad( \pm 0.01)
\end{aligned}
$$




$$
\begin{aligned}
r & =0.920, \mathrm{SD}=0.02, n=15, F=30.33 \\
(\mathrm{R} & =\text { the same as those included in } \mathrm{I}-4)
\end{aligned}
$$

These results suggest that the inclusion of $\sigma_{\mathrm{siA}}$ is indispensable. The correlation coefficient matrices of the 2 series in $\mathrm{CCl}_{4}$ solution are as follows:

a. $\operatorname{BuR}(n=23)$

\begin{tabular}{lrrrr}
\hline & \multicolumn{1}{c}{$\sigma_{i}$} & $\sigma_{s^{\circ}}^{+}$ & $\left(\sigma_{s^{\circ}}^{+}\right)^{2}$ & $\sigma_{\text {si A A }}$ \\
\hline$\sigma_{i}$ & 1.000 & & & \\
$\sigma_{s^{\circ}}^{+}$ & -0.446 & 1.000 & & \\
$\left(\sigma_{s^{\circ}}^{+}\right)^{2}$ & -0.482 & 0.955 & 1.000 & \\
$\sigma_{\text {si A }}$ & 0.479 & 0.053 & -0.049 & 1.000 \\
\hline
\end{tabular}

where substituent $\mathrm{R}=\mathrm{NMe}_{2}, \mathrm{NH}_{2}, \mathrm{OEt}, \mathrm{OMe}, \mathrm{OH}$, tert $-\mathrm{Bu}$, iso-Pr, $\mathrm{Et}, \mathrm{Me}, \mathrm{F}, \mathrm{Cl}, \mathrm{Br}, \mathrm{I}, \mathrm{H}$, $\mathrm{COPh}, \mathrm{COEt}, \mathrm{COMe}, \mathrm{CHO}, \mathrm{CONH}_{2}, \mathrm{COOEt}, \mathrm{COOMe}, \mathrm{CN}, \mathrm{NO}_{2}$.

b. $\operatorname{PrR}(n=20)$

\begin{tabular}{lrrrr}
\hline \hline & \multicolumn{1}{c}{$\sigma_{i}$} & $\sigma_{s^{+}}^{+}$ & $\left(\sigma_{s^{+}}^{+}\right)^{2}$ & $\sigma_{\text {sí }}$ \\
\hline$\sigma_{i}$ & 1.000 & & & \\
$\sigma_{s^{+}}^{+}$ & -0.349 & 1.000 & & \\
$\left(\sigma_{s^{\circ}}^{+}\right)^{2}$ & -0.336 & 0.943 & 1.000 & \\
$\sigma_{\text {si A }}$ & 0.516 & 0.063 & -0.071 & 1.000 \\
\hline
\end{tabular}

where substituent $\mathrm{R}=\mathrm{NH}_{2}, \mathrm{OPr}$, OEt, OMe, OH, tert-Bu, Et, $\mathrm{Cl}, \mathrm{Br}, \mathrm{I}, \mathrm{H}, \mathrm{COPh}, \mathrm{COEt}$, $\mathrm{COMe}, \mathrm{CHO}, \mathrm{CONH}_{2}, \mathrm{COOEt}, \mathrm{COOMe}, \mathrm{CN}, \mathrm{NO}_{2}$.

\section{Conclusion}

The results I-1-III-4 given above afford the following conclusions.

a. Both ${ }^{13} \mathrm{C}$ and ${ }^{1} \mathrm{H}$ SCS (or CS) can be expressed by linear combinations of the entropic and enthalpic contributions.

b. The fall-off of the coefficients of $\sigma_{i}$ at the $\alpha$-, $\beta$ - and $\gamma$-positions are: 138.12, 4.18, -12.82 for ${ }^{13} \mathrm{C}$ and $7.73,1.63,0.34$ for ${ }^{1} \mathrm{H}$ SCS in $\mathrm{CCl}_{4}$ solution; $128.67,5.96,-12.43$ for ${ }^{13} \mathrm{C}$ and $7.59,1.40,0.16$ for ${ }^{1} \mathrm{H} \mathrm{CS}$ in the gas phase.

c. The participation of an entropic term for electron-attracting substituent groups could be ruled out for all ${ }^{13} \mathrm{C}$ and ${ }^{1} \mathrm{H}$ SCS. In contrast, the participation of the entropic term affords a parabola with a maximum among electron-donating substituent groups for $\beta-{ }^{13} \mathrm{C}$ and $\alpha-{ }^{1} \mathrm{H}$ SCS, but gave a minimum for $\gamma-{ }^{13} \mathrm{C} \mathrm{SCS}$. The inversion of the signs of coefficients observed between Eqs. 4 and 13, and between Eqs. 5 and 14 are consistent with the negative correlation between $\alpha-{ }^{13} \mathrm{C}$ and $\gamma-{ }^{13} \mathrm{C}$ SCS.

d. The estimations of chemical shifts at the $\alpha, \beta, \gamma$ positions in both ${ }^{13} \mathrm{C}$ and ${ }^{1} \mathrm{H}$ NMR provided above suggest that the parameters governing chemical shifts of substituted $n$-alkanes in $\mathrm{CCl}_{4}$ solution are the same as those in the vapor phase.

\section{References}

1) Part XVI: Y. Sasaki, H. Takai and T. Tsujimoto, Chem. Pharm. Bull., 28, 3106 (1980).

2) J. A. Pople, J. Chem. Phys., 37, 53, 60 (1962); idem, ibid., 38, 1276 (1963); M. Karplus and J. A. Pople, ibid., 38, 
2803 (1963); J. A. Pople, J. W. McIver, Jr. and N. S. Ostlund, ibid., 49, 2960 (1968); J. A. Pople and D. P. Santry, Mol. Phys., 8, 1 (1964); J. A. Pople, ibid., 7, 269, 301 (1964); G. E. Maciel, J. W. McIver, Jr., O. S. Ostlund and J.

A. Pople, J. Am. Chem. Soc., 92, 1, 11, 4151, 4497, 4506 (1970).

3) Y. Sasaki and M. Suzuki, Chem. Pharm. Bull., 20, 2522 (1972).

4) P. D. Ellis, G. E. Maciel and J. W. McIver, Jr., J. Am. Chem. Soc., 94, 4069 (1971).

5) E. G. Paul and D. M. Grant, J. Am. Chem. Soc., 85, 1701 (1963).

6) J. B. Stothers, "Carbon-13 NMR Spectroscopy," Academic Press, Inc., London, 1972, p. 96.

7) H. Takai, A. Odani and Y. Sasaki, Chem. Pharm. Bull., 27, 1780 (1979).

8) Y. Sasaki, T. Takagi, Y. Yamazato, A. Iwata and H. Kawaki, Chem. Pharm. Bull., 29, 3073 (1981); Y. Sasaki, T. Takagi, A. Iwata and H. Kawaki, unpublished.

9) Y. Sasaki, T. Takagi, H. Kawaki and A. Iwata, Chem. Pharm. Bull., 31, 330 (1983).

10) M. Sawada, M. Ichihara, Y. Yukawa, T. Nakachi and Y. Tsuno, Bull. Chem. Soc. Jpn., 53, 2055 (1980).

11) Y. Tsuno, M. Fujio, M. Sawada and Y. Yukawa, Tetrahedron Lett., 23, 213 (1982).

12) J. R. Cavanaugh and B. P. Dailey, J. Chem. Phys., 34, 1099 (1961).

13) D. R. Stull, E. F. Westrum, Jr. and G. C. Sinke, "The Chemical Thermodynamics of Organic Compounds," John Wiley and Sons, Inc., New York, 1969, and data cited therein; S. W. Benson, "Thermochemical Kinetics," John Wiley and Sons, Inc., New York, 1968; S. W. Benson, F. R. Cruickshank, D. M. Golden, G. R. Haugen, H. E. O'Neal, A. S. Rodger, R. Shaw and R. Walsh, Chem. Rev., 69, 279 (1969).

14) H. C. Brown and G. K. Barbaras, J. Am. Chem. Soc., 75, 6 (1953).

15) H. Otaki, M. Tanaka and S. Hunahashi, "Yoeki Hanno No Kagaku," Gakkai Shuppan Center, 1977 , p. 217.

16) Appleman and Dailey, Adv. Magnet. Reson., 7, 305 (1974).

17) Appleman and Dailey, Adv. Magnet. Reson., 7, 266, 267, 300, 301 (1974).

18) W. Adcock and D. P. Cox, J. Org. Chem., 44, 3004 (1979).

19) Toshio Tsujimoto, Ph.D. Thesis, Osaka Univ., 1981. 